\title{
Quantification of Biomolecular Dynamics Inside Real and Synthetic Nuclear Pore Complexes Using Time-Resolved Atomic Force Microscopy
}

George J. Stanley, ${ }^{\dagger, \#}$ Bernice Akpinar, ${ }^{\dagger, \dagger, \#}$ Qi Shen, ${ }^{\S, \|}$ Patrick D. Ellis Fisher, ${ }^{\S, \| \odot ~ C . ~ P a t r i c k ~ L u s k, ~}{ }^{\S \odot}$ Chenxiang Lin, $\$, \|_{(\infty)}$ and Bart W. Hoogenboom*, ${ }^{\dagger, \perp_{\odot}}$

${ }^{\dagger}$ London Centre for Nanotechnology, University College London, 17-19 Gordon Street, London WC1H 0AH, United Kingdom

*Department of Chemistry, Imperial College London, London SW7 2AZ, United Kingdom

${ }^{\S}$ Department of Cell Biology, Yale School of Medicine, New Haven, Connecticut 06520, United States

"Nanobiology Institute, Yale University, West Haven, Connecticut 06516, United States

${ }^{\perp}$ Department of Physics and Astronomy, University College London, Gower Street, London WC1E 6BT, United Kingdom

Supporting Information

ABSTRACT: Over the past decades, atomic force microscopy (AFM) has emerged as an increasingly powerful tool to study the dynamics of biomolecules at nanometer length scales. However, the more stochastic the nature of such biomolecular dynamics, the harder it becomes to distinguish them from AFM measurement noise. Rapid, stochastic dynamics are inherent to biological systems comprising intrinsically disordered proteins. One role of such proteins is in the formation of the transport barrier of the nuclear pore complex (NPC): the selective gateway for macromolecular traffic entering or exiting the nucleus. Here, we use AFM to observe the dynamics of intrinsically disordered proteins from two systems: the transport barrier

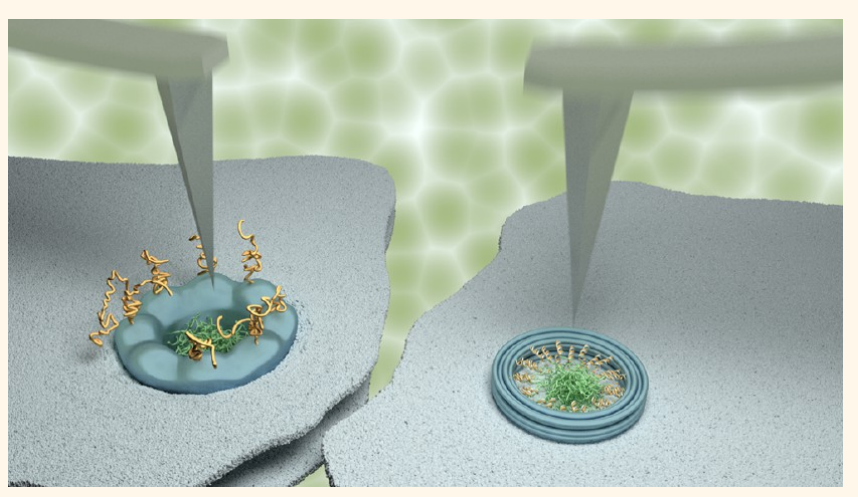
of native NPCs and the transport barrier of a mimetic NPC made using a DNA origami scaffold. Analyzing data recorded with 50-200 ms temporal resolution, we highlight the importance of drift correction and appropriate baseline measurements in such experiments. In addition, we describe an autocorrelation analysis to quantify time scales of observed dynamics and to assess their veracity - an analysis protocol that lends itself to the quantification of stochastic fluctuations in other biomolecular systems. The results reveal the surprisingly slow rate of stochastic, collective transitions inside mimetic NPCs, highlighting the importance of FG-nup cohesive interactions.

KEYWORDS: atomic force microscopy, nuclear pore complex, nucleoporins, intrinsically disordered proteins, nuclear transport, DNA origami

W th its ability to resolve biomolecules in aqueous solution at a spatial resolution of $\sim 1 \mathrm{~nm},{ }^{1}$ atomic force microscopy (AFM) can observe dynamic biological processes at the single-molecule level, without chemical tagging. Thanks to technological advances in microscope hardware, cantilevers, and imaging modes, this high spatial resolution has become compatible with a temporal resolution on the order of $100 \mathrm{~ms}$ per frame (often denoted as high-speed AFM, or HS-AFM), ${ }^{2}$ or $0.5-1 \mathrm{~ms}$ for monitoring individual scan lines. ${ }^{3-5}$ Over the years, AFM has allowed realtime visualization of diverse biological systems, from the binding/unbinding of GroEL molecules ${ }^{3}$ to molecular motors walking along actin filaments, ${ }^{6}$ DNA cleaving by Cas $9,{ }^{7}$ and a range of protein assembly processes ${ }^{4,8-10}$ (among many other applications). ${ }^{2}$

When the observed dynamics are progressive in nature and have a magnitude well above the noise floor of the AFM, they can be unambiguously associated with molecular motion, binding, or conformational change. However, at molecular length scales, such dynamics invariably contain a stochastic

Received: March 28, 2019

Accepted: June 26, 2019

Published: June 26, 2019 

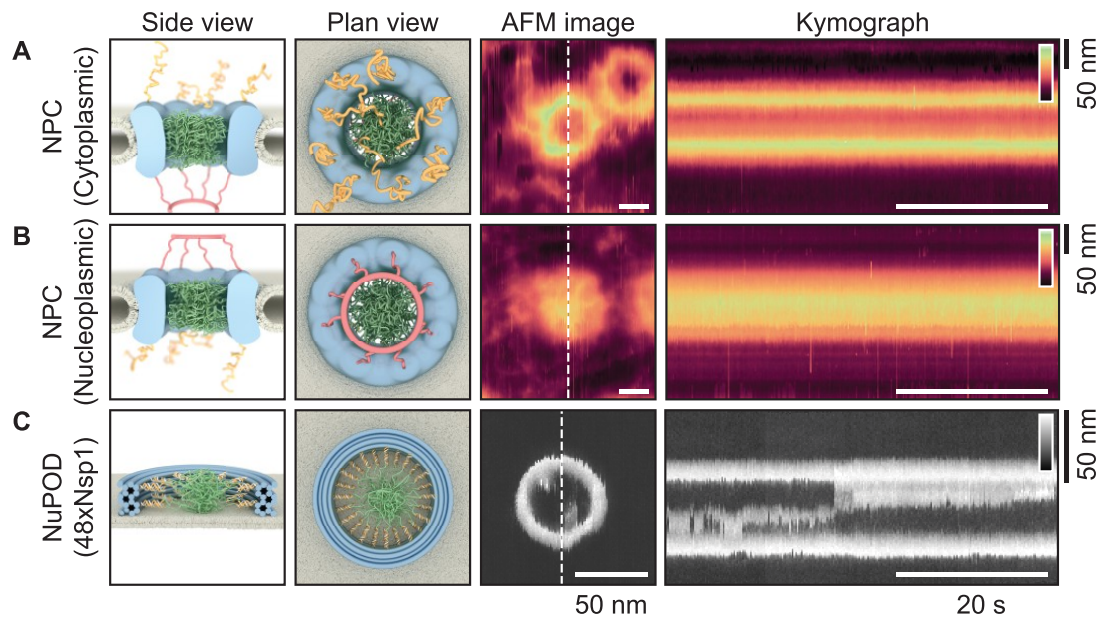

Figure 1. Producing kymographs from Xenopus laevis oocyte NPCs and from NuPODs. Schematics (side and top views) and representative AFM images of NPCs with the cytoplasmic (A) and nucleoplasmic (B) sides facing the AFM tip and of a DNA-origami-based NPC mimic ("NuPOD"; ${ }^{5}$ ) that contains $48 \mathrm{Nsp1}$ proteins, resting on a supported lipid bilayer. The NPC is asymmetrical when viewed through the mirror plane of the nuclear envelope: From the cytoplasmic face, cytoplasmic filaments are seen (yellow filaments), and from the nucleoplasmic face, the nuclear basket protrudes (red filaments). The disordered FG-nups are shown in green (A-C). Kymographs are obtained by recording the same scan line across the pore (white-dashed lines in AFM images) as a function of time (horizontal axis of the kymographs). Note the differences in scale bars between the NPC and NuPOD data. Height scales (insets in kymographs): $80 \mathrm{~nm}$ for the NPCs and $20 \mathrm{~nm}$ for the NuPOD.

component, and when the magnitude of these dynamics is $\lesssim 1$ $\mathrm{nm}$, they are not trivial to distinguish from AFM measurement noise or feedback errors that may arise when tracing molecular contours at high scan speeds. Such stochastic dynamics are arguably most pronounced for natively unfolded and intrinsically disordered proteins. ${ }^{11}$ Unlike structured proteins, intrinsically disordered proteins have a low sequence complexity and fluctuate rapidly over an ensemble of conformations ranging from extended statistical coils to more collapsed globules. Such proteins play a central role in a number of cellular processes: one important example being the selective filtering of macromolecules between the nucleus and the cytoplasm. ${ }^{12-14}$

A large, proteinaceous self-assembly, known as the nuclear pore complex (NPC), controls this flow of materials into and out of the nucleus. The NPC has a cylindrical scaffold that perforates the nuclear envelope, creating a central channel of $\sim 40 \mathrm{~nm}$ in diameter (see Figure 1A,B, schematics). This channel anchors intrinsically disordered proteins enriched with multiple phenylalanine-glycine repeats, known as FG-nucleoporins (or FG-nups). In combination with soluble proteins (nuclear transport receptors; or NTRs), these FG-nups collectively form a selective barrier to nucleocytoplasmic transport. ${ }^{15,16}$ Exactly how they do this remains a topic of debate. $^{14}$

AFM has proven itself as a useful tool for probing the dynamics of FG-nups inside real NPCs, with high-speed experiments potentially revealing dynamic movement of individual FG-nups at the cytoplasmic periphery of the pore ${ }^{15,16}$ and high-resolution studies revealing many different metastable collective morphologies of FG-nups and soluble NTRs inside the central channel. ${ }^{17}$ Both of these results support the notion that the "cohesivity" of the FG-nups is tuned such that the energetics of stable FG-nup morphologies lie near transition states, as has been postulated in computational studies. ${ }^{18-20}$ One of the caveats with these experimental results, however, is that the exact chemical composition of the NPC's transport barrier is ill-defined. Structural studies indicate that, by mass, the FG-nups only comprise (approx- imately) one-third of the material inside the channel; the majority of the pore lumen is filled with NTRs and cargo molecules (i.e., macromolecules bound with NTRs) caught in transit. $^{21}$ Therefore, to better understand the behavior and function of the FG-nups within this complex system, mimetic NPCs made using DNA origami have recently been created. ${ }^{5,22}$

The NuPOD (NucleoPorins Organized on DNA) system provides a platform for studying FG-nup behavior in the cylindrical geometry. It is designed to mimic the dimensions of the NPC central transport channel across species: ${ }^{5}$ The NuPOD inner diameter is $\sim 32-46 \mathrm{~nm}$, in approximate agreement with, for example, the Xenopus laevis transport channel of $\sim 40 \mathrm{~nm}$ inner diameter. ${ }^{17,23}$ Furthermore, the system can accommodate a defined number and type of FGnups. NuPODs therefore contain purely the disordered FGnups of a known composition and grafting density (concentration).

In this study, we use AFM to probe the dynamic behavior of disordered FG-nups across the transport barrier of both real NPCs and mimetic NPCs (NuPODs) containing two different types of purified FG-nup domains from S. cerevisiae, Nsp1 and Nup100, which are orthologues to metazoan Nup62 and Nup98, respectively. For a quantitative comparison of their relative "cohesivity", see refs 19 and 20 . We present analysis, procedures, and criteria to distinguish between the stochastic dynamics of these proteins (often on length scales of $\lesssim 1 \mathrm{~nm}$ ) and the fluctuations due to (also largely stochastic) AFM measurement noise. By applying a drift-correction routine and a subsequent autocorrelation analysis to the pixel heights with time, we can both quantify the time-scale of any dynamic behavior observed and probe the effect of the tip-sample interaction force on the behavior of the system, thus providing a more rigorous approach to interpreting HS-AFM data. In doing so, we reveal the time-scale of collective dynamics of the pure FG-nups inside the NuPOD system, thus further elucidating their precise role in nucleocytoplasmic transport. For simplicity and clarity of representation and for enhanced temporal resolution, we here confine ourselves to kymographs 

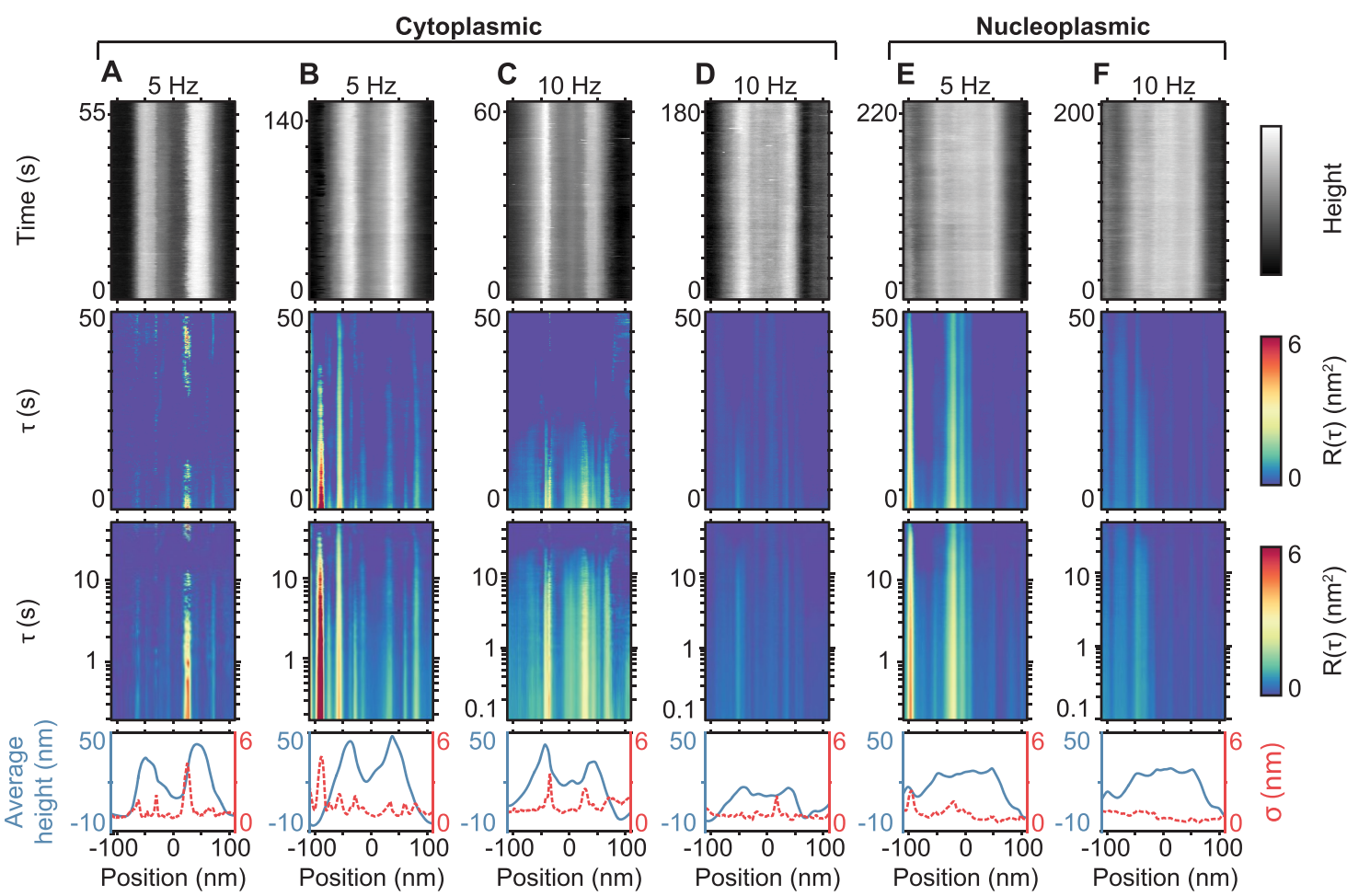

Figure 2. Kymographs and autocorrelation analysis of NPCs. (A-D) Kymographs of NPCs at the cytoplasmic face (top row), recorded as explained in Figure 1, but with time on the vertical axis (recorded at line frequencies of 5 and $10 \mathrm{~Hz}$ ). The concomitant autocorrelation factor, $R$, is displayed as a function of position with respect to the pore center and as a function of time-lag, $\tau$, with $\tau$ plotted on linear (second row) and logarithmic (third row) scales. The average height profiles (blue) and respective standard deviations (red) are plotted in the final row. (E and F) Same as for (A-D) but for the nucleoplasmic face of NPCs. Kymograph height scales: $50 \mathrm{~nm}(\mathrm{~A}$ and C), $60 \mathrm{~nm}(\mathrm{~B})$, $25 \mathrm{~nm}(\mathrm{D}), 40 \mathrm{~nm}$ (E and F). (A-D) Data each from different NPCs. (E and F) The same NPC scanned at 5 and $10 \mathrm{~Hz}$.

acquired by recording single lines across the center of the pores as a function of time (Figure 1 ), ${ }^{3}$ but note that our analysis can also be extended to frame-by-frame recordings (Supplementary Figure 1).

\section{RESULTS AND DISCUSSION}

When acquiring AFM data, temporal resolution can be greatly increased by reducing the dimension of scanning to the fastscan axis only (as was done in this study), thereby producing kymographs. It can be increased further by removing this dimension also and recording the height of a single pixel with time (this has been termed HS-AFM height spectroscopy). ${ }^{4}$ In all types of time-resolved AFM, to accurately measure local height fluctuations as a function of time, lateral drift in the experimental setup must be minimized. Even when using an AFM system with a closed-loop scanner (here: Dimension FastScan, Bruker), in which the sample position is continuously verified to $\sim 1 \mathrm{~nm}$ accuracy, lateral drift still occurs due to scanner hysteresis and thermal equilibration. Therefore, it is only by recording data in at least one dimension spatially (thus rendering points of reference) and employing a drift-correction routine that we can ensure we are recording the same point in space with time. A drift-correction protocol is most accurate when referenced to static objects with steep slopes or protrusions in the data, as this is where a given lateral displacement results in the largest change in the measured AFM signal (i.e., the sample height). Here, all scan lines in a kymograph were aligned laterally by referencing them to the outer edges of the static (and presumably rigid) pore scaffold (see Supplementary Figure 2).
With the kymographs corrected for experimental drift, measured height variations for any given position in the kymograph are, ideally, attributed solely to fluctuations as a function of time. Consequently, the magnitude and time scale of these height fluctuations can be quantified for each (lateral) position by the autocorrelation factor $(R)$ as a function of time-lag $(\tau)$, defined as

$$
R(\tau)=R(m \Delta t) \equiv R_{m}=\frac{1}{N-m} \sum_{n=1}^{N-m}\left[\left(z_{n}-\bar{z}\right)\left(z_{n+m}-\bar{z}\right)\right]
$$

where $m$ and $n$ refer to line numbers in the kymograph, $N$ is the total number of scan lines in the kymograph, $\Delta t$ is the time between consecutive scan lines, $z_{n}$ denotes the pixel height $(\mathrm{nm})$ of a given pixel as a function of time $(n \Delta t)$, and $\bar{z}$ denotes the average pixel height for the (lateral) position under consideration. In brief, $R(\tau)$ quantifies the similarity in height values for a given pixel (in $\mathrm{nm}^{2}$ ) at a certain time lag. It can detect a periodic signal shrouded by noise and the persistence time of stochastically fluctuating signals (see Supplementary Figure 3 for the $R(\tau)$ outputs from various simulated pixel fluctuations). Furthermore, it can be determined for each pixel in a kymograph, and the resultant $R(\tau)$ outputs can be represented as a heatmap as a function of both position (nm) and time-lag $(\tau)$, with red showing greater correlation and blue representing no correlation (see Figure 2).

When this analysis is applied to kymographs produced from the cytoplasmic and nucleoplasmic sides of intact NPCs (Figure 2), at different scan speeds, no significant fluctuations are detected in the positions of the central transport channel 

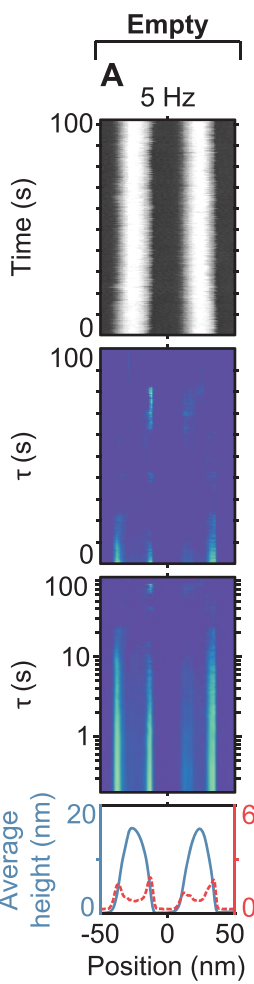
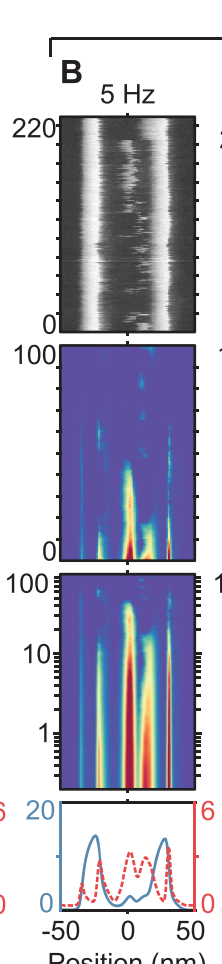

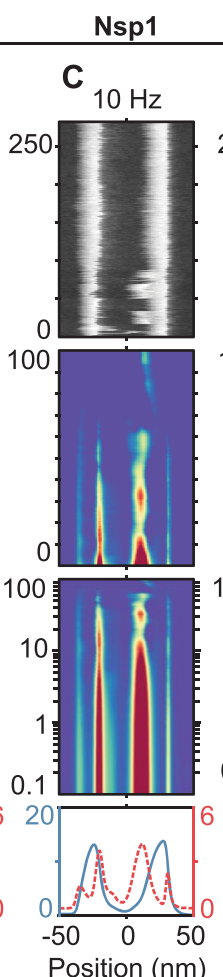

Position (nm)
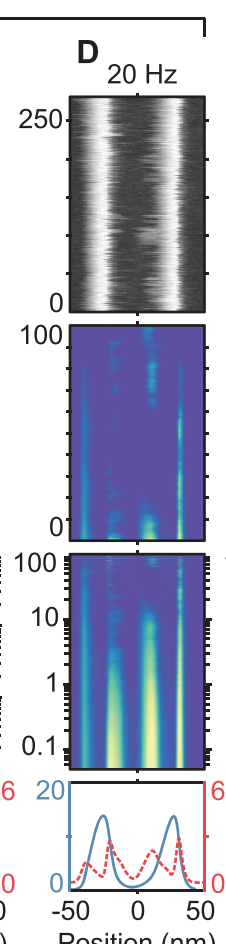

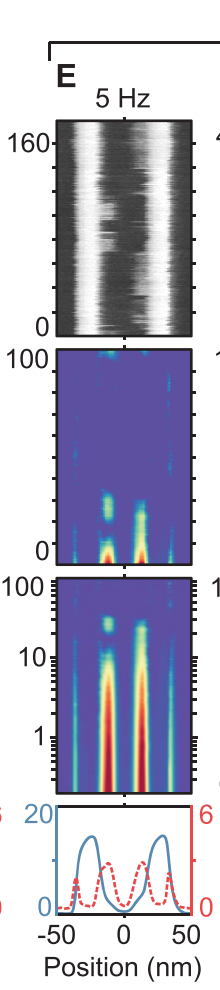

\section{Nup100}
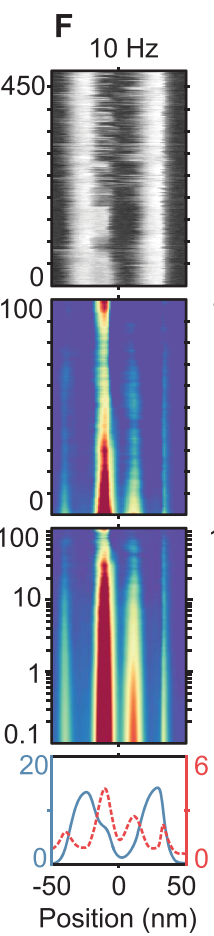
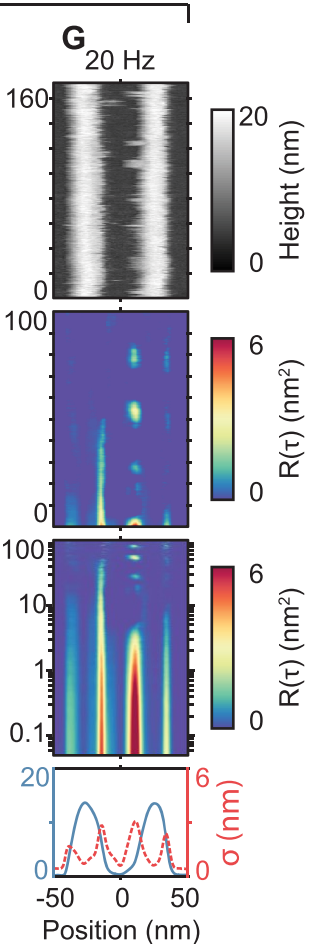

Figure 3. Kymographs and autocorrelation analysis of NuPODs. Repeat of kymograph data, autocorrelation analysis, and averaged height and standard deviation profiles, plotted as in Figure 2, but on NuPOD NPC mimics. Data are shown for an empty NuPOD, that is, bare DNA scaffold without FG-nup domains (A), for NuPODs containing 48 copies of Nsp1 (B-D), and for NuPODs containing 48 copies of Nup100 $(\mathrm{E}-\mathrm{G})$. The data of protein-containing NuPODs were recorded at scan line frequencies of 5,10 , and $20 \mathrm{~Hz}(200,100$, and $50 \mathrm{~ms}$ per scan line). (A-D and G) Data each from different NuPODs. (E and F) The same NuPOD scanned at 5 and $10 \mathrm{~Hz}$.

(i.e., around the $0 \mathrm{~nm}$ position). That is, the recorded fluctuations for positions occupied by the disordered FG-nups are not distinguishable from those at the positions of the nuclear envelope (a double membrane and lamin filaments, here supported by a glass substrate). ${ }^{17}$ It remains possible that FG-nup dynamics inside the central channel yield angströmscale height variations (as have previously been reported for the cytoplasmic face of the transport channel of NPCs), ${ }^{15,16}$ but in our experiments, based on a similar sample preparation, these fluctuations do not exceed the experimental noise floor. This is also apparent from the standard deviation for each position in the kymograph (Figure 2, bottom row).

However, significant fluctuations are detected, in both the $R(\tau)$ heatmaps and standard deviation plots, at positions that coincide with the edges of the NPC scaffold structure. At these edges, lateral drift most strongly affects the detected height (in spite of the applied drift correction, the observed fluctuations at the outer NPC scaffold tend to be larger than the fluctuations detected at the pore center), and the height is more prone to tracking/feedback errors as the AFM tip traces protruding features. Hence, we attribute these fluctuations to artifacts of the AFM imaging process.

Similar artifactual fluctuations are observed when the analysis is applied to the empty pore scaffolds based on the biomimetic NuPOD system (Figure 3A and Supplementary Figure 4). By contrast, when the NuPODs contain grafted FGnups, fluctuations are detected in the pore center that greatly exceed those observed at the same positions in the empty NuPODs or at the background supported lipid bilayer. This is here illustrated for NuPODs each containing a maximum of 48 copies of one of two types of FG-nup domains derived from the $S$. cerevisiae (yeast) NPC (Figure 3B-G; see ref 5 for further details). If $48 \mathrm{Nsp} 1$ molecules are present (Figure 3BD), fluctuating clumps inside the central channel are observed in the kymographs. At both 5 and $10 \mathrm{~Hz}$ (Figure 3B,C, respectively), the autocorrelation heatmaps show a correlation up to $\sim 10 \mathrm{~s}$, while at $20 \mathrm{~Hz}$, little significant correlation is detected beyond $1 \mathrm{~s}$ (see Figure 3D, logarithmic plot). A similar pattern is observed for NuPODs containing 48 Nup100 molecules (Figure $3 \mathrm{E}-\mathrm{G}$ ). At 5 and $10 \mathrm{~Hz}$, correlation is observed up to $\sim 10 \mathrm{~s}$, but at $20 \mathrm{~Hz}$, this appears to be reduced. Furthermore, for all $R(\tau)$ heatmaps, no significant periodicity is detected, and all heatmaps show signals more akin to stochastic fluctuating behavior (see Supplementary Figure 3C and D).

The results presented in Figure 3 are from individual NuPODs. Hence, the results show the stochastic dynamics of a disordered system within a given time frame, resulting in unique kymographs. To assess dynamic behavior over multiple measurements, it is useful to determine ensemble averages. By averaging the kymographs directly, however, the noise would be averaged out. By contrast, the autocorrelation heatmaps can be averaged to build up a picture of the collective FG-nup fluctuations, from many different NuPODs, or merging data recorded over longer times. Furthermore, by cropping only a $30 \mathrm{~nm}$ window, which is smaller than the inner-diameter of the NuPOD $(\sim 32-46 \mathrm{~nm}),{ }^{5}$ it is ensured that only contributions from the FG-nups are considered. This eliminates any artifacts produced by tracking errors from the AFM tip scanning over the DNA scaffold structure. Next, by assuming the NuPODs display rotational symmetry, we can bin the data as a function of radial position, and average. This produces autocorrelation heatmaps as a function of radius, with $0 \mathrm{~nm}$ being the center of 

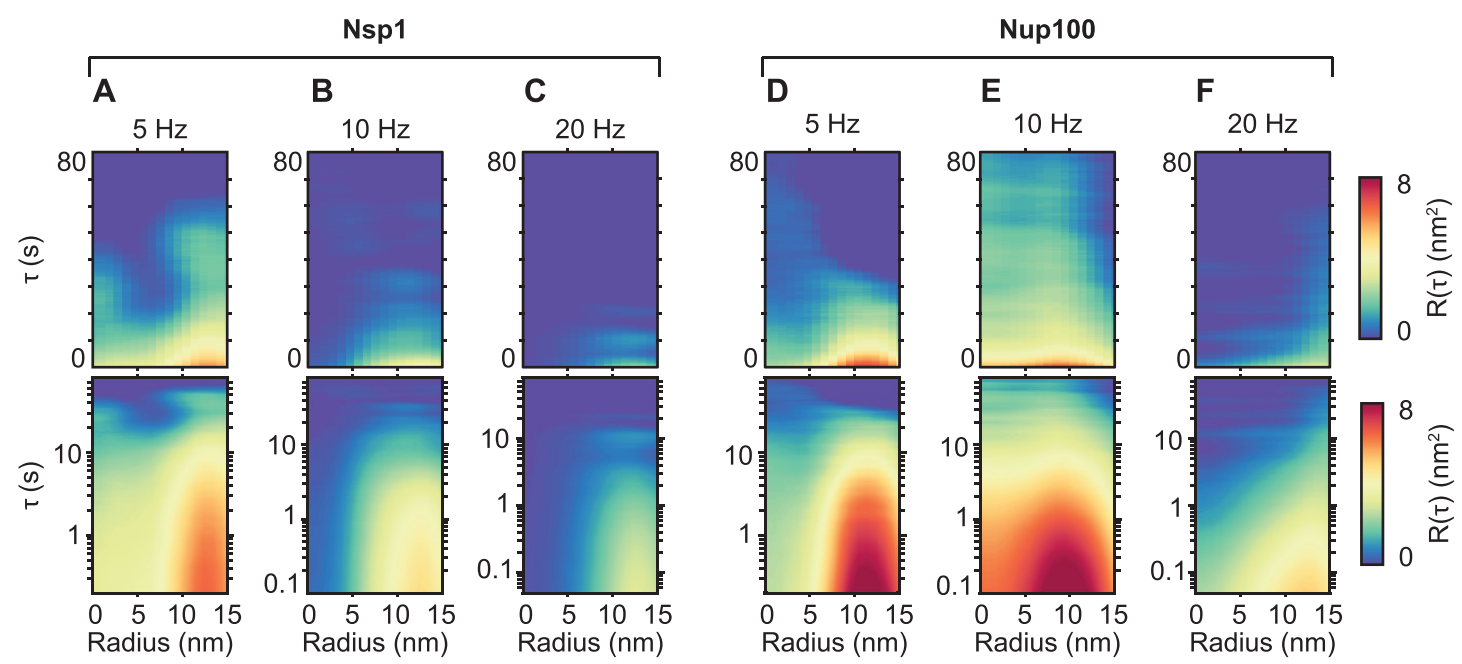

Figure 4. Averaged autocorrelation analysis of the pore channel in NuPODs. $(\mathrm{A}-\mathrm{C}) \mathrm{R}(\tau)$ heatmaps averaged over $n$ NuPODs, each containing $48 \mathrm{Nsp} 1$ molecules, plotted as a function of radial position from the center of the pore, scanned at line rates of 5 , 10, and $20 \mathrm{~Hz} \tau$ is plotted on both linear (top row) and logarithmic scales (bottom row). (D-F) Same as for (A-C) but the NuPODs contain 48 Nup100 molecules. From (A-F): $n=3,4,2,3,5$, and 3; total duration of each kymograph $=739,1050,365,586$, 1907, and 1664 s. It should be noted that one kymograph used in the averaging procedure to produce $(\mathrm{F})$ has previously been published as stand-alone data. ${ }^{5}$
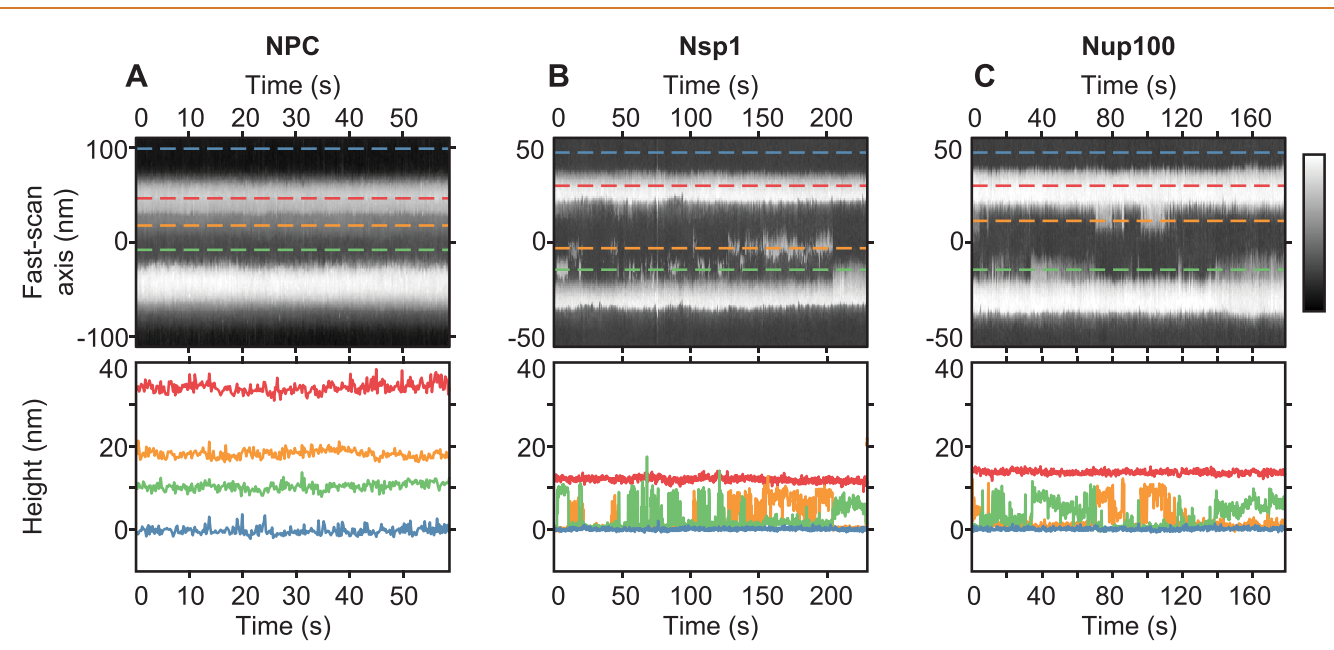

Figure 5. Kymographs and height fluctuations of NPCs and NuPODs. (A) Kymograph of a native NPC (cytoplasmic face) with the height profiles of selected pixels shown underneath, scanned at $5 \mathrm{~Hz}$. Blue-dashed line is the background nuclear envelope; red-dashed line is the NPC scaffold structure; and orange- and green-dashed lines are different locations inside the transport channel. (B and C) Same as for (A) but of NuPODs containing 48 Nsp1 molecules (B) or 48 Nup100 molecules (C). Blue-dashed lines are the background lipid bilayer; reddashed lines are the DNA scaffold structure; and orange- and green-dashed lines are different locations inside the channel. These kymographs are also printed in Figures $2 \mathrm{~A}$ and 3B,E. Color scales: $50 \mathrm{~nm}(\mathrm{~A})$ and $20 \mathrm{~nm}(\mathrm{~B}$ and C).

the channel and $15 \mathrm{~nm}$ approaching the inner-wall of the scaffold. The NuPODs containing 48 Nsp1 molecules (Figure $4 \mathrm{~A}-\mathrm{C})$ exhibit similar behavior to that shown from the individual kymographs (Figure 3B-D), that is, stochastic fluctuations with a decreasing persistence time as the scan rate increases from 5 to 10 to $20 \mathrm{~Hz}$. A very similar picture is seen for the NuPODs containing 48 Nup100 molecules (Figure $4 \mathrm{D}-\mathrm{F})$. At $5 \mathrm{~Hz}$, significant correlation is seen to up to $\sim 10 \mathrm{~s}$ (Figure 4D), and at $10 \mathrm{~Hz}$, it is seen to persist into the $1-10 \mathrm{~s}$ range (Figure 4E). At $20 \mathrm{~Hz}$, however, the correlation does not persist much beyond $1 \mathrm{~s}$ (Figure 4F, logarithmic plot).

By such comparison of autocorrelation analysis of data recorded at different scan speeds, we can also assess the robustness of our results against AFM-dependent (i.e., not intrinsic) parameters. While the data in Figure 4A-F confirm the presence of dynamics for all scan speeds shown here, in general, we observe a decrease in persistence time with increasing scan speed. A plausible explanation is that more aggressive feedback settings are required to track the NuPODs at higher scan speeds (data here were all obtained using tapping mode AFM, with the applied force minimized as much as possible without losing track of the NuPODs). As a consequence, the force sensitivity of the AFM reduces, and the flexible and mobile FG-nups may increasingly be pushed away by the AFM tip. In this context, we note that it is not trivial to quantify the forces applied and energy dissipated into the sample $^{10}$ and that it is near impossible to translate such forces and energies into plausible molecular deformations without the knowledge of the dissipative mechanisms in the sample. It is possible, therefore, that the frequency of FG-nup fluctuations would decrease further in the absence of the AFM tip. 
All six heatmaps (Figure 4A-F) show stochastic, fluctuating behavior (see Supplementary Figure 3C,D) and more correlation at the edges of the transport channels (i.e., nearer radial values of $15 \mathrm{~nm}$ ) as opposed to the center (i.e., at $0 \mathrm{~nm}$ ). Empty NuPOD controls confirm that these fluctuations are related to FG-nup fluctuations (see Supplementary Figure 4). Our observations indicate that the FG-nups spend more time clumped toward the inner-walls of the NuPODs, rather than in the middle of the transport channel, and that their rate of collective transitioning is recorded to increase with faster line scanning. Furthermore, at equal scan speeds, the more cohesive GLFG-repeat Nup100 tends to persist for longer before transitioning when compared with the FxFG-repeat Nsp1.

Taken together, these results show that, while no significant fluctuations are detected inside the native NPC (Figure 2), stochastic changes in height are detected for FG-nups inside the NuPOD system, persisting for up to $\sim 10 \mathrm{~s}$ (when scanned at slower line rates; see Figure $4 A, D$ ). This can be further confirmed by plotting sample height at different locations in and around the pores as a function of time (Figure 5). For the NuPOD system, fluctuations inside the channel exceed those measured on and outside the pore scaffold by more than an order of magnitude (Figure 5B,C), whereas, for the native NPC, no noticeable difference in the magnitude of fluctuations is detected for different positions inside and outside the transport channel (Figure 5A). It is plausible that the discrepancy in behavior observed between the native NPCs and NuPODs (i.e., nonfluctuating and fluctuating behavior) is due to the presence/absence of endogenous NTRs (present in the real NPCs). In the preparation of native nuclear membranes from Xenopus laevis oocytes, the nuclei are repeatedly washed to remove cellular material, including cargoes and NTRs, that may be caught in transit. However, it is safe to assume that many NTRs and possibly cargo molecules bound to the FG-nups will not be removed by our washing steps. ${ }^{21}$ In the case of the NuPODs, in the complete absence of NTRs, and in the symmetry of the pore geometry, there are many different possible metastable conformational states with small activation energy barriers between them, thus leading to the observed fluctuating behavior. However, in the presence of multivalent NTRs (such as importin- $\beta$ ), ${ }^{24}$ the FGnups could wrap around these proteins, thereby becoming energetically trapped in a given conformation (as was observed for FG-nups at low grafting densities in planar films). ${ }^{25}$ To this end, it would be interesting to acquire further kymograph data of NuPODs in the presence of NTRs, presumably, upon addition of importin- $\beta$, FG-nup fluctuations would stop. This experiment was attempted, but the addition of importin- $\beta$ at physiological concentrations $(\sim 1 \mu \mathrm{M})$ disrupted the supported lipid bilayer in our experimental setup.

\section{CONCLUSIONS}

We have shown that purified FG-nups, grafted inside a cylinder, interact collectively to form clumps that persist for $\sim 1$ $\mathrm{s}$ before transitioning. Furthermore, we show a subtle difference in behavior between the FxFG-repeat Nsp1 and the more cohesive GLFG-repeat Nup100, with Nup100 persisting for longer times before transitioning (at comparable scan speeds; see Figure 4). While this result is expected, ${ }^{26}$ it further emphasizes the importance of cohesion-alongside molecular-scale dynamics - to the collective behavior of the FG-nups. For example, Nsp1, which adopts a more extended state than Nup100, ${ }^{6}$ is nevertheless shown to form clumps that can persist for several seconds before transitioning. In the native NPC, however, the static appearance of the FG-nups is probably due to the presence of other macromolecular contents (i.e., NTRs and cargoes) that interact with the FGnups and trap them in given morphologies. ${ }^{17,21}$

More generally, our results highlight several criteria that can be applied to facilitate the interpretation of nanometer-scale fluctuations in time-resolved AFM data, especially of dynamic data obtained from biological systems exhibiting a stochastic, rather than progressive nature. First, data must be background corrected, referenced to stable and flat areas of the sample (as is a common procedure for AFM images), and, separately, corrected for lateral drift. Second, if fluctuations are observed in the vicinity of protruding features on the sample, additional controls are needed (e.g., the empty DNA scaffold control in Figure 3 to rule out any artifactual nature). Third, to rule out white noise, dynamic features should be observed to persist over multiple subsequent scan lines (in unfiltered data), such as observed here in the kymographs recorded on NuPODs, and further articulated by the autocorrelation analysis (Figure 3 ), demonstrating and quantifying the persistence time of the observed molecular dynamics. Fourth, any such fluctuations should be significantly larger than fluctuations at other, presumably immobile positions on the sample (Figure 5). Finally, like any experimental method that probes individual molecules, AFM is an invasive technique. To identify the effect of the tip upon induced molecular dynamics, the observed movements should be shown to be relatively robust against variations in imaging parameters and/or speed (Figure 4).

\section{METHODS}

NuPOD Sample Preparation and AFM Imaging. NuPOD Assembly. DNA origami structures and FG-domains conjugated to DNA were prepared as previously described. ${ }^{5}$ These were stored at -20 and $-80{ }^{\circ} \mathrm{C}$, respectively. The NuPODs were assembled by mixing DNA-labeled FG-nups with terminal MBP-tags in 7.5-fold excess (over the handle number) to DNA origami cylinders in NuPOD buffer $(1 \times$ PBS, $0.1 \%$ Tween $20,10 \%$ glycerol, and $10 \mathrm{mM}$ $\mathrm{MgCl}_{2}$ ). After incubating at $37{ }^{\circ} \mathrm{C}$ for $3 \mathrm{~h}$, the sample was cooled to room temperature, and TEV-protease added to remove the terminal MBP-tag from the FG-nups now conjugated to the DNA rings. After a further incubation for $1.5 \mathrm{~h}, \mathrm{NuPOD}$ were purified from excess protein by ratezonal centrifugation through a glycerol gradient.

Preparation of Lipid Vesicles. 1,2-Dihexadecanoyl-sn-glycero-3phosphocholine (DPPC) and dimethyldioctadecylammonium (bromide salt; DDAB) were purchased from Avanti Polar Lipids. DPPC and $\mathrm{DDAB}$ were mixed at a molar ratio of $3: 1$ in chloroform solvent. Lipid vesicles were prepared by the extrusion method as described elsewhere ${ }^{5}$ and stored at $4{ }^{\circ} \mathrm{C}$ for up to 1 week. Briefly, the chloroform was evaporated under a stream of nitrogen gas to yield a lipid film that was then redispersed with sonication in Milli-Q water. The lipid vesicles were extruded through a $100 \mathrm{~nm}$ polycarbonate filter (GE Healthcare Lifesciences, Buckinghamshire, UK), and the extrusion process was repeated at least 20 times to yield small unilamellar vesicles.

AFM Sample Preparation. $2 \mu \mathrm{L}$ of the lipid vesicles, along with 1 $\mu \mathrm{L}$ of $1 \mathrm{M} \mathrm{MgCl}_{2}, 1 \mu \mathrm{L}$ of $1 \mathrm{M} \mathrm{CaCl}_{2}$, and $16 \mu \mathrm{L}$ of Milli-Q water were deposited onto a freshly cleaved mica disc. The disc was placed in a humid chamber and heated to $65^{\circ} \mathrm{C}$ for $10 \mathrm{~min}$ to induce vesicle rupture. The sample was slowly cooled to room temperature over 20 min to form a gel-phase supported lipid bilayer (SLB). Excess vesicles in the supernatant were removed by rinsing first with water and then exchanged to imaging buffer (10 mM PB $26 \mathrm{mM} \mathrm{MgCl}_{2}, \mathrm{pH} 7.0$ ). The rinsing process was repeated three to five times to ensure a clean and uniform surface before addition of $2-4 \mu \mathrm{L}$ of NuPODs $(\sim 2-5$ 
$\mathrm{nM})$ prior to imaging. The DNA origami scaffold is electrostatically adsorbed to the positively charged membrane.

AFM Imaging. All AFM measurements were performed at room temperature in liquid. Images were obtained using a Dimension FastScan Bio AFM (Bruker) operated in Tapping mode. FastScan D (Bruker) cantilevers were used for all imaging with a resonance frequency of $\sim 110 \mathrm{kHz}$, measured spring constant of $\sim 0.15 \mathrm{~N} \mathrm{~m}^{-1}$, and quality factor of $\sim 2$ in water. The force applied to the sample was minimized by setting the highest possible amplitude set-point voltage, which was typically above $85 \%$ of free oscillation close to the sample surface. Single line scanning experiments provided an enhanced time resolution while minimizing disturbance to the NuPODs. For these experiments, a single NuPOD was centered and the frame size decreased to $120 \mathrm{~nm}$ before disabling the slow-scan axis over the center of the pore. Where possible, data were collected at 5,10 , and $20 \mathrm{~Hz}$ for each pore imaged.

Nuclear Envelope Sample Preparation and AFM Imaging. Xenopus laevis Oocyte Nuclear Envelope Preparation. All experiments were conducted on Xenopus laevis oocyte NPCs. The oocytes were stored in modified Barth's solution $(88 \mathrm{mM} \mathrm{NaCl}, 15 \mathrm{mM}$ Tris, $2.4 \mathrm{mM} \mathrm{NaHCO}_{3}, 0.82 \mathrm{mM} \mathrm{MgCl}_{2}, 1 \mathrm{mM} \mathrm{KCl}, 0.77 \mathrm{mM} \mathrm{CaCl}_{2}$, and $\mathrm{U} / 100 \mu \mathrm{g}$ penicillin/streptomycin, $\mathrm{pH} 7.4$ ) at $4{ }^{\circ} \mathrm{C}$ for a maximum of 3 days. The nuclei were isolated, and the nuclear envelopes were prepared, in nuclear isolation medium (NIM) buffer $(10 \mathrm{mM} \mathrm{NaCl}$, $90 \mathrm{mM} \mathrm{KCl}, 10 \mathrm{mM} \mathrm{MgCl}, 10 \mathrm{mM}$ Tris, $\mathrm{pH}$ 7.4), for AFM imaging as previously described. ${ }^{17}$ The nuclei were kept in buffer and on ice throughout the entire sample preparation, and no chemical fixation or detergent was used at any stage.

AFM Imaging. All AFM measurements were performed at room temperature in import buffer $\left(20 \mathrm{mM}\right.$ Hepes, $110 \mathrm{mM} \mathrm{CH}_{3} \mathrm{COOK}, 5$ $\mathrm{mM} \mathrm{Mg}\left(\mathrm{H}_{3} \mathrm{COO}\right)_{2}, 0.5 \mathrm{mM}$ EGTA, $\mathrm{pH}$ 7.4). Kymographs were obtained using a Dimension FastScan Bio AFM (Bruker), using tapping mode AFM. FastScan D (Bruker) cantilevers were used for all experiments, and the applied force was minimized (optimized) as described for the NuPODs. Images of the nuclear envelope were recorded to ascertain if the AFM tip probed the cytoplasmic or nucleoplasmic face of the nuclear envelope. ${ }^{17}$ A $300 \times 300 \mathrm{~nm}$ image at 304 samples/line of a single NPC was recorded; this ensured capture of background nuclear envelope as well as the NPC. When the position along the slow-scan axis was at the center of the NPC (see Figure 1A,B, AFM images, white-dashed lines), the slow scan-axis movement was disabled. A kymograph was then produced with height in the fast-scan axis and time in the slow-scan axis. The line rate was set to 5,10 , or $20 \mathrm{~Hz}$, and the gains were optimized to best track the contours of the sample.

Analysis Protocols. All data analyses were done using MATLAB (MathWorks). The analysis code is accessible from GitHub: https:// github.com/geostanley/AFM---Kymographs---Autocorrelation--DriftCorrection.

Concatenating Kymographs. A sequence of kymographs recorded from one pore (NuPOD or NPC), at a given line rate, was loaded into MATLAB, and the kymograph width (nm), samples/line, and line rate $(\mathrm{Hz})$ were entered manually. A first-order plane background subtraction was applied to each kymograph individually to flatten the data with respect to the background (either nuclear envelope or lipid bilayer). If the Down Scan Only feature during data capture was not enabled, the capture direction of the first kymograph was entered (up or down). If the Down Scan Only feature was not enabled, the script vertically flipped intermittent kymographs. If the Down Scan Only feature was enabled, this step was skipped. All images were then vertically concatenated. This yielded one large kymograph with height in the fast-scan axis and time (as captured chronologically) in the slow-scan axis. A first-order plane background subtraction was applied to the concatenated kymograph, again using the background nuclear envelope or lipid bilayer.

Drift Correction of Kymographs. The concatenated kymograph was shown as a figure. The two outer edges of the scaffold (for either the NuPOD or NPC) were selected manually. Windows (usually $\sim 10-20 \mathrm{~nm}$ in width) of kymograph data, centered around the two selected points, were cropped. The height data within these two windows were averaged to create a template. Each line (fast-scan axis) within the kymograph, at the same window positions, was then compared with the template using the sum of absolute differences (SAD) method, that is, the sum of absolute difference in height values between all relevant pixels was calculated, with a lower SAD score meaning greater correlation. Each line (or height array) was shifted in $1 \mathrm{~nm}$ intervals to both the left and right by half the window width. The SAD score was calculated at each position. Whichever position gave the lowest SAD score was considered to have the best correlation with the template, and the height array was moved to this position. If shifted, however, this resulted in missing data points at one end of the height array (if a row was shifted $6 \mathrm{~nm}$ to the left, for example, there would be $6 \mathrm{~nm}$ of missing data points at the extreme right end of the array). As it is assumed that this region is either background lipid bilayer (for NuPODs) or background nuclear envelope (for NPCs), these pixels were filled in with random noise between the values of -1 and $1 \mathrm{~nm}$. Schematics of this routine, for both the NPC and NuPOD, can be seen in Supplementary Figure 2.

Autocorrelation Analysis. After drift correction, an autocorrelation function was applied to the kymograph. This was defined as in eq 1 in the Results and Discussion section. It should be noted that it is possible to normalize $R(\tau)$ values by dividing by the pixel's variance $\left(\sigma^{2}\right)$. This would give $R(\tau)$ values between -1 and 1 , at each time-lag, for each pixel, in which -1 is perfect anticorrelation and 1 is perfect correlation. However, to facilitate a quantitative comparison between experiments (in which all will have different $\sigma^{2}$ values for each pixel), this was not done. The $R(\tau)$ values were calculated for each pixel for $\tau$ $=\Delta t$ to $\tau=100 \mathrm{~s}$ (or until the maximum time of the kymograph if recorded for $<100 \mathrm{~s}$ ), in increments of $\Delta t$.

To average autocorrelation heatmaps, at a given line rate, from several NuPODs, the heatmaps must be aligned spatially. This was done by selecting the two inside edges of the scaffold structure as seen in the concomitant kymograph (akin to the drift correction routine, in which both outer edges are selected). These two values were used to define the center of the kymograph (and thereby the center of their autocorrelation heatmaps). All autocorrelation heatmaps were then aligned by this point, and the $R(\tau)$ values $15 \mathrm{~nm}$ either side of this position were cropped. All heatmaps to be averaged were then scaled by their overall time contribution and then summed. The data were then binned as a function of radial position and averaged to produce an autocorrelation heatmap as a function of radius, with $0 \mathrm{~nm}$ being the center of the channel and $15 \mathrm{~nm}$ approaching the inner-wall of the scaffold (see Figure 4).

\section{ASSOCIATED CONTENT}

\section{S Supporting Information}

The Supporting Information is available free of charge on the ACS Publications website at DOI: 10.1021/acsnano.9b02424.

Image sequences of an NPC and a NuPOD containing 48 Nsp1 molecules (Supplementary Figure 1); a schematic of the drift-correction algorithm (Supplementary Figure 2); example autocorrelation outputs applied to various simulated signals (Supplementary Figure 3); and results showing the autocorrelation analysis applied to the empty NuPOD controls (Supplementary Figure 4) (PDF)

\section{AUTHOR INFORMATION}

\section{Corresponding Author}

*E-mail: b.hoogenboom@ucl.ac.uk.

\section{ORCID}

Patrick D. Ellis Fisher: 0000-0002-4248-108X

C. Patrick Lusk: 0000-0003-4703-0533

Chenxiang Lin: 0000-0001-7041-1946

Bart W. Hoogenboom: 0000-0002-8882-4324 


\section{Author Contributions}

"These authors contributed equally to this work. G.J.S. designed and performed NPC experiments, developed analysis software, performed analysis, and wrote the manuscript. B.A. designed and performed NuPOD experiments and wrote the manuscript. P.D.E.F. and Q.S. prepared the DNA rings and purified protein constructs for NuPOD experiments. C.P.L. and C.L. conceptualized the NuPOD system. B.W.H. conceptualized the experiments and analysis and wrote the manuscript. All authors reviewed and commented on the manuscript and its intellectual content.

\section{Notes}

The authors declare no competing financial interest.

\section{ACKNOWLEDGMENTS}

The authors acknowledge Ariberto Fassati (University College London) for useful discussions, Alice Pyne (University College London) for training and advice on AFM, and the Yale Institute for Nanoscience and Quantum Engineering for use of AFM equipment. This work was funded by UK BBSRC and EPSRC studentships (BB/J014567/1 for G.J.S.; EP/L015277/ 1, EP/L504889/1 for B.A.), EPSRC equipment funding (EP/ M028100/1), and by the NIH (R21GM109466 for Q.S. and C.P.L.).

\section{REFERENCES}

(1) Pyne, A.; Thompson, R.; Leung, C.; Roy, D.; Hoogenboom, B. W. Single-Molecule Reconstruction of Oligonucleotide Secondary Structure by Atomic Force Microscopy. Small 2014, 10, 3257-3261.

(2) Dufrêne, Y. F.; Ando, T.; Garcia, R.; Alsteens, D.; MartinezMartin, D.; Engel, A.; Gerber, C.; Müller, D. J. Imaging Modes of Atomic Force Microscopy for Application in Molecular and Cell Biology. Nat. Nanotechnol. 2017, 12, 295-307.

(3) Viani, M. B.; Pietrasanta, L. I.; Thompson, J. B.; Chand, A.; Gebeshuber, I. C.; Kindt, J. H.; Richter, M.; Hansma, H. G.; Hansma, P. K. Probing Protein-Protein Interactions in Real Time. Nat. Struct. Biol. 2000, 7, 644-647.

(4) Heath, G. R.; Scheuring, S. High-Speed AFM Height Spectroscopy Reveals Ms-Dynamics of Unlabeled Biomolecules. Nat. Commun. 2018, 9, 4983.

(5) Fisher, P. D. E.; Shen, Q.; Akpinar, B.; Davis, L. K.; Chung, K. K. H.; Baddeley, D.; Šarić, A.; Melia, T. J.; Hoogenboom, B. W.; Lin, C.; Lusk, C. P. A Programmable DNA Origami Platform for Organizing Intrinsically Disordered Nucleoporins within Nanopore Confinement. ACS Nano 2018, 12, 1508-1518.

(6) Kodera, N.; Yamamoto, D.; Ishikawa, R.; Ando, T. Video Imaging of Walking Myosin $\mathrm{V}$ by High-Speed Atomic Force Microscopy. Nature 2010, 468, 72-76.

(7) Shibata, M.; Nishimasu, H.; Kodera, N.; Hirano, S.; Ando, T.; Uchihashi, T.; Nureki, O. Real-Space and Real-Time Dynamics of CRISPR-Cas9 Visualized by High-Speed Atomic Force Microscopy. Nat. Commun. 2017, 8, 1430.

(8) Chiaruttini, N.; Redondo-Morata, L.; Colom, A.; Humbert, F.; Lenz, M.; Scheuring, S.; Roux, A. Relaxation of Loaded ESCRT-III Spiral Springs Drives Membrane Deformation. Cell 2015, 163, 866879.

(9) Leung, C.; Hodel, A. W.; Brennan, A. J.; Lukoyanova, N.; Tran, S.; House, C. M.; Kondos, S. C.; Whisstock, J. C.; Dunstone, M. A.; Trapani, J. A.; Voskoboinik, I.; Saibil, H. R.; Hoogenboom, B. W. Real-Time Visualization of Perforin Nanopore Assembly. Nat. Nanotechnol. 2017, 12, 467.

(10) Nievergelt, A. P.; Banterle, N.; Andany, S. H.; Gönczy, P.; Fantner, G. E. High-Speed Photothermal off-Resonance Atomic Force Microscopy Reveals Assembly Routes of Centriolar Scaffold Protein SAS-6. Nat. Nanotechnol. 2018, 13, 696-701.
(11) Miyagi, A.; Tsunaka, Y.; Uchihashi, T.; Mayanagi, K.; Hirose, S.; Morikawa, K.; Ando, T. Visualization of Intrinsically Disordered Regions of Proteins by High-Speed Atomic Force Microscopy. ChemPhysChem 2008, 9, 1859-1866.

(12) Stewart, M. Molecular Mechanism of the Nuclear Protein Import Cycle. Nat. Rev. Mol. Cell Biol. 2007, 8, 195-208.

(13) Wente, S. R.; Rout, M. P. The Nuclear Pore Complex and Nuclear Transport. Cold Spring Harbor Perspect. Biol. 2010, 2, a000562.

(14) Stanley, G. J.; Fassati, A.; Hoogenboom, B. W. Biomechanics of the Transport Barrier in the Nuclear Pore Complex. In Seminars in cell and developmental biology; Elsevier: Exeter, UK, 2017; Vol. 68, pp 4251.

(15) Sakiyama, Y.; Mazur, A.; Kapinos, L. E.; Lim, R. Y. H. Spatiotemporal Dynamics of the Nuclear Pore Complex Transport Barrier Resolved by High-Speed Atomic Force Microscopy. Nat. Nanotechnol. 2016, 11, 719-723.

(16) Mohamed, M. S.; Kobayashi, A.; Taoka, A.; WatanabeNakayama, T.; Kikuchi, Y.; Hazawa, M.; Minamoto, T.; Fukumori, Y.; Kodera, N.; Uchihashi, T.; Ando, T.; Wong, R. W. High-Speed Atomic Force Microscopy Reveals Loss of Nuclear Pore Resilience as a Dying Code in Colorectal Cancer Cells. ACS Nano 2017, 11, 55675578.

(17) Stanley, G. J.; Fassati, A.; Hoogenboom, B. W. Atomic Force Microscopy Reveals Structural Variability amongst Nuclear Pore Complexes. Life Sci. Alliance 2018, 1, No. e201800142.

(18) Osmanovic, D.; Bailey, J.; Harker, A. H.; Fassati, A.; Hoogenboom, B. W.; Ford, I. J. Bistable Collective Behavior of Polymers Tethered in a Nanopore. Phys. Rev. E 2012, 85, 061917.

(19) Zahn, R.; Osmanović, D.; Ehret, S.; Araya Callis, C.; Frey, S.; Stewart, M.; You, C.; Görlich, D.; Hoogenboom, B. W.; Richter, R. P. A Physical Model Describing the Interaction of Nuclear Transport Receptors with FG Nucleoporin Domain Assemblies. eLife 2016, 5, No. e14119.

(20) Vovk, A.; Gu, C.; Opferman, M. G.; Kapinos, L. E.; Lim, R. Y. H.; Coalson, R. D.; Jasnow, D.; Zilman, A. Simple Biophysics Underpins Collective Conformations of the Intrinsically Disordered Proteins of the Nuclear Pore Complex. eLife 2016, 5, No. e10785.

(21) Kim, S. J.; Fernandez-Martinez, J.; Nudelman, I.; Shi, Y.; Zhang, W.; Raveh, B.; Herricks, T.; Slaughter, B. D.; Hogan, J. A.; Upla, P.; Chemmama, I. E.; Pellarin, R.; Echeverria, I.; Shivaraju, M.; Chaudhury, A. S.; Wang, J.; Williams, R.; Unruh, J. R.; Greenberg, C. H.; Jacobs, E. Y.; et al. Integrative Structure and Functional Anatomy of a Nuclear Pore Complex. Nature 2018, 555, 475.

(22) Ketterer, P.; Ananth, A. N.; Laman Trip, D. S.; Mishra, A.; Bertosin, E.; Ganji, M.; van der Torre, J.; Onck, P.; Dietz, H.; Dekker, C. DNA Origami Scaffold for Studying Intrinsically Disordered Proteins of the Nuclear Pore Complex. Nat. Commun. 2018, 9, 902.

(23) Eibauer, M.; Pellanda, M.; Turgay, Y.; Dubrovsky, A.; Wild, A.; Medalia, O. Structure and Gating of the Nuclear Pore Complex. Nat. Commun. 2015, 6, 7532.

(24) Isgro, T. A.; Schulten, K. Binding Dynamics of Isolated Nucleoporin Repeat Regions to Importin- $\beta$. Structure 2005, 13, 1869-1879.

(25) Lim, R. Y. H.; Fahrenkrog, B.; et al. Nanomechanical Basis of Selective Gating by the Nuclear Pore Complex. Science 2007, 318, 640-644.

(26) Yamada, J.; Phillips, J. L.; Patel, S.; Goldfien, G.; CalestagneMorelli, A.; Huang, H.; Reza, R.; Acheson, J.; Krishnan, V. V.; Newsam, S.; Gopinathan, A.; Lau, E. Y.; Colvin, M. E.; Uversky, V. N.; Rexach, M. F. A Bimodal Distribution of Two Distinct Categories of Intrinsically Disordered Structures with Separate Functions in FG Nucleoporins. Mol. Cell. Proteomics 2010, 9, 2205-2224. 